Pacific Journal of Mathematics

TOPISMS AND INDUCED NON-ASSOCIATIVE SYSTEMS

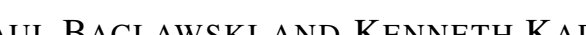




\title{
TOPISMS \\ AND \\ INDUCED NON-ASSOCIATIVE SYSTEMS
}

\author{
Kenneth BaClawski and Kenneth M. KapP
}

A triple of bijections $(\gamma ; \alpha, \beta)$ from one binary system onto another is called an isotopism if the maps transfer the multiplication: $(x y)^{r}=x^{\alpha} y^{\beta}$. These maps have been used by Albert, Bruck and others in the study of quasigroups and loops. A natural generalization of isotopism is given to a triple of maps called a topism, which transfers just the multiplication, as above, from one binary system into another. It will be shown, (1.7), that a topism from one quasigroup into another is an isotopism if and only if any one of the maps is one-to-one and onto.

Two maps, $\alpha, \beta$ on a binary system $(A, \cdot)$ induce a new binary operation, $\circ$, defined in the natural fashion $x \circ y=$ $x^{\alpha} y^{\beta}$. The relationship between topic imbeddings and induced systems is studied in $\S 2$. It is shown, (2.3), that one groupoid is topically imbeddable in another precisely when it is isomorphic to a subgroupoid of an induced groupoid of the second. Thus, (2.7), two quasigroups are isotopic if and only if one is isomorphic to an induced groupoid of the other.

Finally, the imbeddings of nonassociative binary systems into semigroups and groups are considered. It is shown, (3.1) and (3.2), that groupoids can be imbedded as ideals in semigroups. However, it is seen (3.4) that a groupoid with identity is topically imbeddable in a group precisely when the groupoid is isomorphic to a subsemigroup, with identity, of the given group. From this a generalization, (3.6), of Albert's Theorem that a loop is isotopic to a group if and only if they are isomorphic is obtained.

1. Preliminaries; topic imbedding.

Definition 1.1. Let $(A, \cdot)$ and $(B, \circ)$ be two groupoids. An ordered triple $(\gamma ; \alpha, \beta)$ of maps $\gamma, \alpha, \beta$ from $A$ into $B$ will be called a topism from $A$ into $B$ if $(x \cdot y)^{\gamma}=x^{\alpha} \circ y^{\beta}$ for all $x, y \in A$.

2. An isotopy from $A$ onto $B$ is a topism in which all three maps are one-to-one and onto.

3. A topism $(\gamma ; \alpha, \beta)$ is said to be one-to-one or onto when $\gamma$ is one-to-one or onto respectively. 
When there is no confusion as to the binary operation of a given groupoid, we will often write the product of two elements as a simple juxtaposition.

Definition 1.2. Let $A, B$ and $C$ be groupoids. If $(\gamma ; \alpha, \beta)$ and $(\nu ; \lambda, \mu)$ are topisms from $A$ into $B$ and from $B$ into $C$ their product is defined by the usual functional composition:

$$
(\gamma ; \alpha, \beta) \circ(\nu ; \lambda, \mu)=(\gamma \circ \nu ; \alpha \circ \lambda, \beta \circ \mu) \text {. }
$$

The following proposition is readily verified.

Proposition 1.3. Let $(\gamma ; \alpha, \beta)$ be a topism from a groupoid $A$ into a groupoid $B$ and $(\nu ; \lambda, \mu)$ a topism from $B$ into a groupoid $C$. The product $(\gamma \circ \nu ; \alpha \circ \lambda, \beta \circ \mu)$ is a topism from $A$ into $C$ which is one-to-one if both $\gamma$ and $\nu$ are one-to-one and onto if both $\gamma$ and $\nu$ are onto.

For any two groupoids $A$ and $B$ it always is possible to define a topism from one into the other: select $b, b^{\prime} \in B$ and define $A^{\alpha}=\{b\}$, $A^{\beta}=\left\{b^{\prime}\right\}$ and $A^{\gamma}=\left\{b b^{\prime}\right\}$. One readily checks that these constant maps define a topism $(\gamma ; \alpha, \beta)$ of $A$ into $B$. Our preliminary concern is to find necessary and sufficient conditions for the existence of a oneto-one topism from one groupoid into or onto another. As might be expected we make the following:

Definition 1.4. Let $(A, \cdot)$ and $(B, \circ)$ be two groupoids. We say that $(A, \cdot)$ is topically imbeddable in $(B, \circ)$ if there exists a one-to-one topism $(\gamma ; \alpha, \beta)$ fom $A$ into $B$.

Corollary 1.5. Let $A, B$ and $C$ be groupoids. If $A$ is topically imbeddable in $B$ and if $B$ is topically imbeddable in $C$ then $A$ is topically imbeddable in $C$.

REMARK AND ExAmple 1.6. While isotopy induces an equivalence relation, the same is not true for topic imbeddability. Although reflexive and transitive it is not necessarily symmetric, as the following example shows. Let $A=\{a, b\}=B$ and let the products be defined in $(A, \cdot)$ and $(B, \circ)$ by the Cayley tables:

\begin{tabular}{c|c|c|}
$\cdot$ & $a$ & $b$ \\
\hline$a$ & $a$ & $a$ \\
\hline$b$ & $a$ & $a$
\end{tabular}

\begin{tabular}{c|c|c|}
$\circ$ & $a$ & $b$ \\
\hline$a$ & $a$ & $b$ \\
\hline$b$ & $b$ & $b$ \\
\hline
\end{tabular}


Then $A$ is a null semigroup with one nonzero element $b$ while $B$ is a cyclic group of order 2 with $a$ as the identity.

Define $\gamma=1: A \rightarrow B$ and $A^{\alpha}=A^{\beta}=\{\alpha\}$. One readily checks that $(\gamma ; \alpha, \beta)$ is a topism from $A$ into $B$, and an imbedding onto $B$ since $\gamma$ is one-to-one and onto. On the other hand there is no topic imbedding $(\nu ; \lambda, \mu)$ from $B$ into $A$ since $\nu$ must be constant: we have for any $x, y \in B: a=x^{\lambda} \cdot y^{\mu}=(x \circ y)^{\nu}$ but $a \circ b=b, a \circ a=a$ and $\nu$ is not one-to-one.

That topic imbeddability is not too far removed from the usual concept of isotopy is shown by the following:

THeORem 1.7. Let $A$ and $B$ be quasigroups. A topism $(\gamma ; \alpha$, $\beta$ ) from $A$ into $B$ is an isotopy if and only if any one of the maps $\gamma, \alpha$ or $\beta$ is one-to-one and onto.

Proof. The theorem can be proven in a straightforward manner. The following approach was suggested by the referee to whom we express our thanks.

Let $(\gamma ; \alpha, \beta)$ be a topism from $(A, \circ)$ into $(B, \cdot)$. Define (cf. [9], $[4$, p. 8,9$])$ the conjugate quasigroups $(A, *),(B,+)$ by

$$
\begin{array}{lr}
x \circ y=z<=>y=x * z & x, y, z \in A \\
u \cdot v=w<=>v=u+w & u, v, w \in B .
\end{array}
$$

(1) The equation, $z^{r}=(x \circ y)^{\gamma}=x^{\alpha} y^{3}$

(2) transforms into, $z^{r}=x^{\alpha} \cdot(x * z)^{3}$

(3) and then into, $x^{\alpha}+z^{\gamma}=(x * z)^{3}$.

Suppose now that $\beta$ is one-to-one and onto. Fix $x=a \in A$ in (2) to give

$$
z^{\gamma}=a^{\alpha} \cdot(a * z)^{\beta} .
$$

Multiplication by a fixed element, in a quasigroup, is one-to-one and onto, so $\gamma$ is one-to-one and onto. Equation (3) shows that if $\gamma$ is one-to-one and onto then so is $\beta$.

Similarly it may be shown that $\alpha$ is one-to-one and onto if and only if $\gamma$ is one-to-one and onto.

The converse follows immediately from the definition of isotopy. The theorem is thus proved.

Careful observation of the above proof shows that the assumptions of one-to-one and onto are independent. Thus the following corollary is immediate. 
Corollary 1.8. Let $A$ and $B$ be quasigroups. A topism $(\gamma ; \alpha$, $\beta$ ) from $A$ into $B$ is an imbedding if and only if anyone of the maps $\gamma, \alpha$ or $\beta$ is one-to-one; it is onto if and only if anyone of the maps $\gamma, \alpha$ or $\beta$ is onto.

Proposition 1.9. Let $A$ be a quasigroup and $B$ a finite groupoid. There exists a topic imbedding of $A$ onto $B$ if and only if $A$ and $B$ are isotopic. In such a case $B$ is also a quasigroup.

Proof. Let us assume that $(\gamma ; \alpha, \beta)$ is a topic imbedding of $A$ onto $B$, i.e., $\gamma$ is one-to-one and onto. If for $b_{\imath} \in A$ we have $b_{1}^{\beta}=b_{2}^{\beta}$ then with any $a_{0} \in A$ it follows that $\left(a_{0} b_{1}\right)^{\gamma}=a_{0}^{\alpha} b_{1}^{\beta}=a_{0}^{\alpha} b_{2}^{\beta}=\left(a_{0} b_{2}\right)^{\gamma}$. Hence $a_{0} b_{1}=a_{\vec{v}} b_{2}$ and since $A$ is a quasigroup we have $b_{1}=b_{2}$. Thus $\beta$ is one-to-one. We have $|A|=|B|<\infty$ since $\gamma$ is one-to-one and onto and hence $\beta$ is immediately onto. In a similar fashion $\alpha$ is oneto-one and onto. Thus $(\gamma ; \alpha, \beta)$ is an isotopy and by Bruck [4, III, 2 , ii] we have that $B$ is also a quasigroup. The converse is obvious.

REMarK 1.10. Consider the groupoids of example (1.6). Define a triple of maps $(\gamma ; \alpha, \beta)$ from $B$ into $A$ as follows: $B^{\gamma}=B^{\alpha}=\{\alpha\}$ and $\beta=1: B \rightarrow A$. Since any product in $A$ is a it is clear that $(\gamma$; $\alpha, \beta)$ is a topism. Here $\beta$ is one-to-one and onto, $B$ a (quasi) group. Thus, unlike the case (1.7), (1.9) will not necessarily hold with assumptions on anyone of the maps $\gamma, \alpha$ or $\beta$.

2. Topic imbeddability and induced systems. In this section we will investigate the relationship between topic imbeddability of one groupoid into another and the induced groupoids of a given groupoid.

Definition 2.1. Let $(A, \cdot)$ be a groupoid and $\alpha, \beta$ mappings of $A$ into itself. The groupoid $\left(A\right.$, ०) with $x \circ y=x^{\alpha} \cdot y^{\beta}$ for $x$, $y \in A$ is called the groupid of $A$ induced by $\alpha$ and $\beta$ and is denoted by $(A, \circ)=(A, \cdot ; \alpha, \beta)$.

If $A$ is a groupoid we will speak of an induced groupoid of $A$ when the maps $\alpha$ and $\beta$ on $A$ are either implicit from the context or are to be determined.

Proposition 2.2. Every induced groupoid of a given groupoid $(A, \cdot)$ is topically imbeddable in $(A, \cdot)$.

Proof. Let $(A, \circ)=(A, \cdot ; \alpha, \beta)$ be an induced groupoid of $A$. Then $\left(1_{A} ; \alpha, \beta\right)$ is a topic imbedding of $(A, \circ)$ onto $(A, \cdot)$ since 
$(x \circ y)^{1}{ }^{1}=x \circ y=x^{\alpha} y^{\beta}$.

Theorem 2.3. A groupoid $(A, \cdot)$ is topically imbeddable in a groupoid $(B, *)$ if and only if $(A, \cdot)$ is isomorphic to a subgroupoid of an induced groupoid of $(B, *)$.

Proof. Let $(\nu ; \lambda, \mu)$ be a topic imbedding of $A$ into $B$. Define maps $\lambda^{\prime}$ and $\mu^{\prime}$ on $B$ by:

$$
x^{\lambda^{\prime}}=\left\{\begin{array}{ll}
x^{\nu^{-1} \lambda} & \text { if } x \in A^{\nu} \\
x & \text { if } x \notin A^{\nu}
\end{array} \quad y^{\mu^{\prime}}= \begin{cases}y^{\nu-1}{ }^{\prime} & \text { if } y \in A^{\nu} \\
y & \text { if } y \notin A^{\nu} .\end{cases}\right.
$$

Consider $(B, \circ)=\left(B, * ; \lambda^{\prime}, \mu^{\prime}\right)$. For $x, y \in A$ we have

$$
(x \cdot y)^{\nu}=x^{\lambda} * y^{\mu}=\left(x^{\nu}\right)^{\nu^{-1} \lambda} *\left(y^{\nu}\right)^{\nu-1 \mu}=\left(x^{\nu}\right)^{\lambda^{\prime}} *\left(y^{\nu}\right)^{\mu^{\prime}}=x^{\nu} \circ y^{\nu} .
$$

Thus $\nu$ is an isomorphism from $(A, \cdot)$ onto a subgroupoid $A^{\nu}$ of $(B, \circ)$, an induced groupoid of $(B, *)$.

Conversely, let us suppose that $\nu$ is an isomorphism from $(A, \cdot)$ into a subgroupoid $B^{\prime}$ of an induced groupoid $(B, \circ)=(B, * ; \alpha, \beta)$ of $B$. Then $\lambda=\nu \alpha$ and $\mu=\nu \beta$ are maps from $A$ into $B$ and we have $(x \cdot y)^{\nu}=x^{\nu} \circ y^{\nu}=x^{\nu \alpha} * y^{\nu \beta}=x^{\lambda} * y^{\mu}$. Thus $(\nu ; \lambda, \mu)$ is an imbedding into $(B, *)$. The proof is now complete.

REMARK 2.4. We see above that when $A^{\nu} \neq B$ we have complete freedom in defining $\alpha$ and $\beta$ on $B \backslash A^{\nu}$. Thus even if a groupoid $A$ is topically imbeddable in a groupoid $B$, the induced groupid of $B$ in (2.3) is not uniquely determined.

Definition 2.5. The induced groupoid $(B, \circ)=\left(B, * ; \quad \lambda^{\prime}, \mu^{\prime}\right)$ defined in (2.3) will be called the naturally induced groupoid of $B$ relative to the topic imbedding $(\nu ; \lambda, \mu)$.

The next corollary readily follows from the preceding proof.

COROLlary 2.6. Let $A$ and $B$ be two groupoids. Then there is a topic imbedding of $A$ onto $B$ if and only if $A$ is isomorphic to an induced groupoid of $B$.

We can combine this with (1.7) and (1.8) to obtain:

COROLlary 2.7. Let $A$ and $B$ be quasigroups. Then $A$ is isotopic to $B$ if and only if $A$ is isomorphic to an induced groupoid of $B$. 
We observe from (1.6), considering $\alpha$ and $\beta$ as maps on $B=A$, that, while $(B, \circ)$ is a group, the induced groupoid $(A, \cdot)=(B, \circ ; \alpha, \beta)$ is far from being a quasigroup. However it is possible to determine necessary and sufficient conditions for the induced groupoid of a quasigroup to be a quasigroup.

Proposition 2.8. Let $(A, \cdot)$ be a quasigroup. The induced groupoid $(A, \circ)=(A, \cdot ; \alpha, \beta)$ of $A$ is a quasigroup if and only if both $\alpha$ and $\beta$ are one-to-one and onto.

Proof. (The following approach was kindly suggested by the referee.) The induced groupoid $(A, \circ)$ of the quasigroup $(A, \cdot)$ is defined by $x \circ y=x^{\alpha} y^{\beta}$ or equivalently $(x \circ y)^{\gamma}=x^{\alpha} y^{\beta}$ where we take $\gamma=1_{A}$, the identity map on $A$. Clearly $(\gamma ; \alpha, \beta)$ is a topic imbedding from $(A, \circ)$ onto $(A, \cdot)$. Thus by $(1.7)$ if $(A, \circ)$ is a quasigroup then $\alpha$ and $\beta$ are one-to-one and onto since $\gamma$ is one-to-one and onto.

Conversely if $\alpha$ and $\beta$ are one-to-one and onto, then $\left(1_{A} ; \alpha, \beta\right)$ is a principal isotopy $([4, \mathrm{p} .56])$ from $(A, \circ)$ onto $(A, \cdot)$. It follows that $(A, \circ)$ is a quasigroup since isotopy is an equivalence relation on the class of groupoids and every isotope of a quasigroup is a quasigroup $([4$, p. 56]).

We can combine (2.7) and (2.8), since the isomorphic image of a quasigroup is a quasigroup, to obtain the topism $\left(1_{B} ; \alpha, \beta\right)$, which Bruck ([4, p. 56]) calls a principal isotopism of $B$ (when the maps $\alpha$ and $\beta$ are one-to-one and onto as in (2.8)) and the following result which contains his remark $([4, \mathrm{p} .56])$ on principal isotopes:

Corollary 2.9. Two quasigroups $(A, \cdot)$ and $(B, \circ)$ are isotopic if and only if $A$ is isomorphic to some principal isotope, $(B, \circ ; \alpha, \beta)$, (for $\alpha, \beta$ one-to-one and onto maps) of $B$.

3. Topic imbeddings into semigroups and groups. In this section we will investigate the topic imbeddability of groupoids into semigroups and groups. We will give two imbeddings for the first problem; in the first imbedding the image is a two-sided ideal in a commutative semigroup, in the second the image is a one-sided ideal of a transformation semigroup and is similar to the usual Cayley construction.

THEOREM 3.1. Every groupoid is topically imbeddable in some commutative semigroup. Indeed the semigroup and topism $(\gamma ; \alpha, \beta)$, 
can be chosen such that the image under $\gamma$ is a two-sided ideal in the semigroup and in the naturally induced groupoid of the semigroup.

Proof. Let $(A, \cdot)$ be a groupoid and let $S=A x\{1,2,3\}$. Let $z \in A$ be fixed. Define a product $*$ in $S$ as follows: Let

$$
(x, i) *(y, j)= \begin{cases}(x y, 1) & \text { if } i=2, j=3 \\ (y x, 1) & \text { if } i=3, j=2 \\ (z, 1) & \text { otherwise. }\end{cases}
$$

One quickly checks the following properties of $(S, *)$ :

(1) $S * S \leqq A \times\{1\}$

(2) $S *(A \times\{1\})=(A \times\{1\}) * S=\{(z, 1)\}$

(3) $(S * S) * S \subseteq(A \times\{1\}) * S=\{(z, 1)\}$

(4) $S *(S * S) \subseteq S *(A \times\{1\})=\{(z, 1)\}$ and

(5) $(x, i) *(y, j)=(y, j) *(x, i)$.

From (3) and (4) it is immediate that $(S, *)$ is associative and commutativity follows directly from (5).

For $x \in A$ define $x^{\gamma}=(x, 1), x^{\alpha}=(x, 2)$ and $x^{\beta}=(x, 3)$. We readily check for $x, y \in A:(x y)^{r}=(x y, 1)=(x, 2) *(y, 3)=x^{\alpha} * y^{\beta}$ so that $(\gamma ; \alpha, \beta)$ is a topism. Obviously each of the maps $\gamma, \alpha$ and $\beta$ is one-to-one. Furthermore, since $S * S \subseteq A \times\{1\}=A^{r}$ the image of $A$ under $\gamma$ is a two-sided ideal in $(S, *)$ and in the naturally induced groupoid $\left(S, * ; \alpha^{\prime}, \beta^{\prime}\right)$ of $S$.

The images of $A$ in the semigroup under the maps $\gamma, \alpha$ and $\beta$ above are disjoint. In the next theorem from a given groupoid, $A$, we construct a Cayley-like semigroup $S$, and topic imbedding $(\gamma ; \gamma, \beta)$ into $S$ so that the image $A^{r}$ is a right-ideal in $\left(S, * ; \gamma^{\prime}, \beta^{\prime}\right)$.

THEOREM 3.2. Let $(A, \cdot)$ be a groupoid. Then there exists a semigroup $(S, *)$ and a topic imbedding $(\gamma ; \gamma, \beta)$ of $A$ into $S$ such that $A^{r}$ is a minimal ideal in $S$ but only a right ideal in the naturally induced groupoid $\left(S, * ; \gamma^{\prime}, \beta^{\prime}\right)$.

Proof. Let $R=\left[\left\{\rho_{a}: a \in A\right\}\right]$ be the closure of the right multiplications, $\rho_{a}$, determined by elements of $A$ in $\mathscr{T}_{A}$, the transformation semigroup on $A$. Clearly $R$ is a semigroup under the usual functional composition, $\circ$ Let $S=A \cup R$ and define an operation * on $S$ by cases as follows:

$$
\begin{array}{ll}
a * b=b & \text { for } a, b \in A \text { and } \\
\sigma * b=b & \sigma, \tau \in R
\end{array}
$$




$$
\begin{aligned}
& a * \tau=(a) \tau \\
& \sigma * \tau=\sigma \circ \tau .
\end{aligned}
$$

One readily checks in a straightforward manner that $(S, *)$ is associative. For example,

$$
a *(\sigma * \tau)=a *(\sigma \circ \tau)=(a)[\sigma \circ \tau]=((a) \sigma) \tau=(a * \sigma) \tau=(a * \sigma) * \tau
$$

and

$$
(a * b) * \tau=b * \tau=(b) \tau=a *(b) \tau
$$

since

$$
\text { (b) } \tau \in A
$$

We now define $\gamma$ to be the inclusion mapping $\gamma: A \stackrel{i}{\longrightarrow} S$ and $\beta$ the mapping of $A$ into $S$ defined by $a^{\beta}=\rho_{a}$. Let $\alpha=\gamma$. We check for $a, b \in A:(a \cdot b)^{\gamma}=a \cdot b=(a) \rho_{b}=a * \rho_{b}=a^{\alpha} * b^{\beta}$. Since $\gamma$ is oneto-one it follows that $(\gamma ; \gamma, \beta)$ is a topic imbedding of $A$ into $S$. From the definition of $*$ it is clear that $A^{\gamma}$ is a minimal ideal in $S$.

Now consider the naturally induced groupoid $(S, \circ)=\left(S, * ; \gamma^{\prime}, \beta^{\prime}\right)$ of $(S, *)$. Here, for $s, t \in S$ we have by definition $s \circ t=s^{\prime \prime} * t^{\beta \prime}$ where $\gamma^{\prime}=\gamma$ on $A^{\gamma} \subseteq S$ so that $\gamma^{\prime}=1_{S}$ while $\beta^{\prime}=\beta$ on $A^{\gamma}=A$ and $\beta^{\prime} \mid R=1_{R}$. We readily check the three pertinent products, where $a, b \in A$ and $\sigma, \tau \in R$ :

(i ) $\quad a \circ b=a^{i^{\prime}} * b^{\beta^{\prime}}=a^{\gamma} * b^{\beta}=a * \rho_{b}=(a) \rho_{b} \in A$

(ii) $\quad a \circ \tau=a^{r^{\prime}} * \tau^{\beta^{\prime}}=a^{r} * \tau^{\beta}=a * \tau=(a) \tau \in A$

(iii) $\sigma \circ b=\sigma^{\gamma^{\prime}} * b^{\beta^{\prime}}=\sigma * b^{\beta}=\sigma * \rho_{b}=\sigma \rho_{b} \notin A$

to see that $A$ is a right ideal but not a left ideal. The proof is now complete.

We observe here that $A^{r}$ is a minimal right ideal in $\left(S, * ; \gamma^{\prime}, \beta^{\prime}\right)$ whenever $R$ is transitive on the set $A$. The above proof goes through unaltered if we take $R=\mathscr{T}_{A}$ so that this result is always possible if desired.

The results of $\S 1$ and $\S 2$ were concerned with one-to-one topic images and topic imbeddings of a binary system of a given type into another. We have seen that an arbitrary groupoid can be topically imbedded as an ideal in a semi-group. The remainder of this section deals with the reciprocal question as to what properties a binary system must have if it is imbeddable in another system of a more particular type. Theorem (3.4) and Corollary (3.6) are generalizations of Theorem 2 in Albert's paper on quasigroups [2].

We have the following proposition which is a partial converse to (1.9).

Proposition 3.3. Let $A$ be a groupoid with identity e. There. 
exists an imbedding of $A$ onto some quasigroup $B$ if and only if $A$ and $B$ are isotopic, in which case $A$ is a loop.

Proof. Suppose $(\gamma ; \alpha, \beta)$ is the imbedding of $A$ onto $B$. Then $a^{\gamma}=(a e)^{\gamma}=a^{\alpha} e^{\beta}$ and since in a quasigroup two elements, $\left(a^{\gamma}, e^{\beta}\right)$, in an equation uniquely determine the third, it readily follows that $\alpha$ is one-to-one and onto. In a similar fashion $\beta$ is one-to-one and onto. Thus $A$ and $B$ are isotopic. Since the isotope of a quasigroup is a quasigroup it follows that $A$ is a loop.

THEOREM 3.4. A groupoid with identity is topically imbeddable in a group if and only if the groupoid is isomorphic to a subsemigroup, with identity, of the group and in such a case the groupoid is a semigroup.

Proof. Let $A$ be a groupoid with identity $e$ and let $(\gamma ; \alpha, \beta)$ be a topic imbedding of $A$ into a group $G$. Let $a, b \in A$. Then we have $a^{\gamma}=(a e)^{\gamma}=a^{\alpha} e^{\beta}$ and $b^{\gamma}=(e b)^{\gamma}=e^{\alpha} b^{\beta}$ from which it follows that $a^{\alpha}=a^{\gamma}\left(e^{\beta}\right)^{-1}$ and $b^{\beta}=\left(e^{\alpha}\right)^{-1} b^{\gamma}$. Hence

$$
(a b)^{r}=a^{\alpha} b^{3}=a^{\gamma}\left(e^{3}\right)^{-1}\left(e^{\alpha}\right)^{-1} b^{r}=a^{\gamma}\left(e^{r}\right)^{-1} b^{\gamma} .
$$

Now define $x^{\eta}=x^{r}\left(e^{r}\right)^{-1}$ for $x \in A$. Thus

$$
(a b)^{\eta}=(a b)^{\gamma}\left(e^{\gamma}\right)^{-1}=\left[a^{\gamma}\left(e^{\gamma}\right)^{-1} b^{\gamma}\right]\left(e^{r}\right)^{-1}=\left[a^{\gamma}\left(e^{\gamma}\right)^{-1}\right]\left[b^{\gamma}\left(e^{\gamma}\right)^{-1}\right]=a^{\eta} b^{\eta}
$$

so that $\eta$ is a homomorphism. Since $\gamma$ and right multiplication by $\left(e^{i}\right)^{-1}$ are both one-to-one maps $\eta$ is also one-to-one and thus an isomorphism of $A$ into $G$. The image $(A) \eta$ in $G$ is closed and hence a semigroup. The result follows since associativity is preserved under isomorphisms $\left(\eta^{-1}:(A) \eta \rightarrow A\right)$. Clearly $e^{\eta}=e^{\gamma}\left(e^{\gamma}\right)^{-1}=1$ is the identity of $G$.

The converse is trivial.

The natural inclusion maps $(i=\gamma, \alpha, \beta)$ of the nonnegative integers in the additive group of integers precludes sharpening the semigroup conclusion above. Moreover as the following examples show the assumption that the groupoid have an identity element in both (3.3) and (3.4) is crucial.

EXAMPLES 3.5. Let $A=\{0,1,2\}$ be a null semigroup with three elements, all products equal 0 . Let $\gamma$ be the identity map from the set $A$ onto $B=Z_{3}$ the cyclic group of three elements. For $a \in A$ define $a^{\alpha}=1$ and $a^{\beta}=2$. Then for

$$
x, y \in A: 0^{\gamma}=0=1+2=x^{\alpha}+y^{\beta}
$$


so that $(\gamma ; \alpha, \beta)$ is topic imbedding of $A$ onto $B$ and clearly $A$ is not a quasigroup.

Now let $(A, \cdot)$ be the groupoid defined by the following Cayley table and let $G=Z_{3}=\{0,1,2\}$ be the cyclic group of three elements. Define the maps $\gamma, \alpha, \beta$ from $A$ onto $G$ as follows:

\begin{tabular}{c|c|c|c}
$\cdot$ & $a$ & $b$ & $c$ \\
\hline$a$ & $b$ & $a$ & $c$ \\
\hline$b$ & $c$ & $b$ & $a$ \\
\hline$c$ & $a$ & $c$ & $b$ \\
\hline
\end{tabular}

(a) $\gamma=0,(b)_{r}=1$,

(c) $\gamma=2, \alpha=\gamma,(a) \beta=1$,

(b) $\beta=0$ and (c) $\beta=2$.

A straightforward check shows that $(\gamma ; \alpha, \beta)$ is a topism and since each map is one-to-one and onto $(\gamma ; \alpha, \beta)$ is an isotopy. But we have $(a b) a=b \neq c=a(b a)$ so that $A$ is not associative.

Theorem 3.4 is easily strengthened if the imbedding map $\gamma$ is onto since $\eta=\rho_{(e r)}-1$ is onto also.

COROLlary 3.6. There exists a topic imbedding of a groupoid with identity onto a group if and only if the groupoid is itself a group and in such a case the groups are isomorphic.

\section{REFERENCES}

1. J. Aczel, Conditions for a loop to be a group and for a groupoid to be a semigroup, Amer. Math. Month. 76 (1969), 547-549.

2. A. A. Albert, Quasigroups, I., Trans. Amer. Math. Soc. 54 (1943), 507-519.

3. Otakar Boruvka, Grundlagen der Gruppoid-und Gruppen-theorie, Hochschulbucher fur Mathematik, Bd. 46 (VEB Deutscher Verlag der Wissenchaften, Berlin, 1960).

4. R. H. Bruck, A Survey of Binary Systems, Ergebnisse der Math. Neue Folge, Vol. 20, Springer, 1958.

5. A. H. Clifford and G. B. Preston, The Algebraic Theory of Semigroups, Vol. 1, 2, Math. Survey 7, Amer. Math. Soc., 1961, 1967.

6. P. H. Doyle and R. J. Warne, Some properties of groupoids, Amer. Math. Monthly 70 (1963), 1051-1057.

7. B. A. Hausman and O. Ore, Theory of quasigroups, Amer. J. Math. 59 (1937), 983-1004.

8. Kenneth M. Kapp, Green's Lemma for groupoids (to appear Rocky Mountain J. of Math.)

9. Sherman K. Stein, On the foundations of quasigroups, Trans. Amer. Math. Soc. 85 (1957), 228-256.

Received February 25, 1970. Part of this research was done while the first author received support under a University of Wisconsin-Milwaukee summer undergraduate research program for the summer of 1969.

UNIVERSITY OF WISCONSIN-MILWAUKEE

MilwaUkeE, Wisconsin 


\title{
PACIFIC JOURNAL OF MATHEMATICS
}

\author{
EDITORS
}

H. SAMELSON

Stanford University

Stanford, California 94305

C. R. Новву

University of Washington

Seattle, Washington 98105
J. DUGUNDJI

Department of Mathematics

University of Southern California

Los Angeles, California 90007

RICHARD ARENS

University of California

Los Angeles, California 90024

\section{ASSOCIATE EDITORS}

E. F. BECKENBACH

B. H. NeUManN

F. WOLF

K. YosHidA

\section{SUPPORTING INSTITUTIONS}

UNIVERSITY OF BRITISH COLUMBIA CALIFORNIA INSTITUTE OF TECHNOLOGY UNIVERSITY OF CALIFORNIA MONTANA STATE UNIVERSITY UNIVERSITY OF NEVADA NEW MEXICO STATE UNIVERSITY OREGON STATE UNIVERSITY UNIVERSITY OF OREGON OSAKA UNIVERSITY UNIVERSITY OF SOUTHERN CALIFORNIA
STANFORD UNIVERSITY UNIVERSITY OF TOKYO UNIVERSITY OF UTAH WASHINGTON STATE UNIVERSITY UNIVERSITY OF WASHINGTON

AMERICAN MATHEMATICAL SOCIETY CHEVRON RESEARCH CORPORATION TRW SYSTEMS

NAVAL WEAPONS CENTER 


\section{Pacific Journal of Mathematics}

\section{Vol. 36, No. $1 \quad$ November, 1971}

Norman Larrabee Alling, Analytic and harmonic obstruction on

nonorientable Klein surfaces ............................ 1

Shimshon A. Amitsur, Embeddings in matrix rings .............. 21

William Louis Armacost, The Frobenius reciprocity theorem and essentially bounded induced representations ....................... 31

Kenneth Paul Baclawski and Kenneth Kapp, Topisms and induced

non-associative systems ............................ 45

George M. Bergman, The index of a group in a semigroup ............ 55

Simeon M. Berman, Excursions above high levels for stationary Gaussian

processes....................................... 63

Peter Southcott Bullen, A criterion for $n$-convexity .............. 81

W. Homer Carlisle, III, Residual finiteness of finitely generated commutative

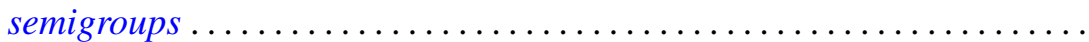

Roger Clement Crocker, On the sum of a prime and of two powers of

two ............................................ 103

David Eisenbud and Phillip Alan Griffith, The structure of serial rings . . . 109

Timothy V. Fossum, Characters and orthogonality in Frobenius

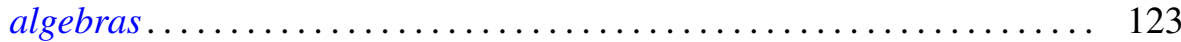

Hugh Gordon, Rings of functions determined by zero-sets . .......... 133

William Ray Hare, Jr. and John Willis Kenelly, Characterizations of Radon partitions...

Philip Hartman, On third order, nonlinear, singular boundary value

problems

David Michael Henry, Conditions for countable bases in spaces of

countable and point-countable type ..

James R. Holub, Hilbertian operators and reflexive tensor products ...

Robert P. Kaufman, Lacunary series and probability ..... . .

195

Erwin Kreyszig, On Bergman operators for partial differential equations in

two variables ................................

Chin-pi Lu, Local rings with noetherian filtrations . .

Louis Edward Narens, A nonstandard proof of the Jordan curve theorem...

S. P. Philipp, Victor Lenard Shapiro and William Hall Sills, The Abel summability of conjugate multiple Fourier-Stieltjes integrals. .

Joseph Earl Valentine and Stanley G. Wayment, Wilson angles in linear normed spaces

Hoyt D. Warner, Finite primes in simple algebras ...

Horst Günter Zimmer, An elementary proof of the Riemann hypothesis for an elliptic curve over a finite field... 